\title{
COVERS OF NON-ALMOST-FINITE TYPE SOFIC SYSTEMS
}

\author{
SUSAN WILLIAMS
}

(Communicated by R. Daniel Mauldin)

\begin{abstract}
An almost finite type (AFT) sofic system $S$ has a cover which intercepts every other cover of $S$ [BKM]. We show that if an irreducible sofic system $S$ is not AFT, it has an infinite collection of covers such that no two are intercepted by a common cover of $S$.
\end{abstract}

0. Introduction. If $\pi$ is a factor map from a subshift of finite type $\Sigma$ onto a sofic system $S$, we call $(\Sigma, \pi)$ - or, for brevity, $\pi$ - a cover of $S$. If $(\Sigma, \pi)$ and $(\Gamma, \phi)$ are covers of $S$ such that $\phi=\pi \circ \theta$ for some factor map $\theta$ from $\Gamma$ to $\Sigma$, we say $\pi$ intercepts $\phi$; if $\theta$ is invertible we say the covers $\phi$ and $\pi$ are conjugate over $S$.

It was shown in [BKM] that an irreducible sofic system $S$ is almost finite type (AFT) if and only if it has a cover which intercepts all other covers of $S$. The authors asked whether a non-AFT sofic system has a finite collection $C$ of covers such that every cover is intercepted by a cover in $C$. In [W] we proved by example that this is not always the case. In this paper we show that there is never such a collection for a non-AFT sofic system.

The example in [W] uses an extremely simple sofic system taken from [BKM]. A feeling that this example is somehow archetypal for non-AFT systems led to the general result. This feeling is made concrete in a characterization of AFT systems which we state as Theorem 2 .

From this point we work to mimic the construction in [W] of an infinite collection of covers such that no two have a common intercepting cover. To do this in a general setting requires some technical groundwork, found in $\S 3$. The construction itself is given in $\S 4$.

1. Properties of the joint cover. For background on subshifts of finite type (SFT) and sofic systems we refer the reader to [AM, BKM, and BMT].

We regard a subshift of finite type $\Sigma$ as being determined by a directed graph $G(\Sigma)$, with the alphabet of $\Sigma$ equal to the edge set of $G(\Sigma)$. Every cover of a sofic system $S$ is conjugate over $S$ to a 1-block cover; that is, a cover $(\Sigma, \pi)$ where $\pi$ is generated by a labeling of the edges of $G(\Sigma)$ with elements of the alphabet of $S$. Such a cover is right resolving if distinct edges in $G(\Sigma)$ with the same initial vertex have distinct labels, and right closing if for some $n$, any two paths in $G(\Sigma)$ with the same initial vertex and same labeling must coincide except on their final $n$ edges. Left resolving and left closing are defined analogously. A word $w$ of $S$ is a

Received by the editors October 6, 1987. Part of this paper was presented at the American Mathematical Society meeting in Laramie, Wyoming, in August, 1985.

1980 Mathematics Subject Classification (1985 Revision). Primary 28D20; Secondary 54H20, 05 C20.

This work was partially supported by a grant from the University of South Alabama Research and Public Service Council. 
resolving word for $(\Sigma, \pi)$ if for some $k$ between 1 and the length of $w$, all paths in $G(\Sigma)$ labeled by $w$ have the same $k$ th edge.

For a word $w$ appearing in a sofic system $S$, we denote by $F(w)$ the set of words $v$ such that $w v$ is a word of $S$ (the follower set of $w$ ) and by $P(w)$ the set of words $u$ with $u w$ in $S$ (the predecessor set of $w$ ). We call $w$ magic if whenever $u w$ and $w v$ appear in $S$, so does $u w v$; equivalently, $F(u w)=F(w)$ for all $u$ in $P(w)$, or $P(w v)=P(w)$ for all $v$ in $F(w)$. Every extension of a magic word is magic.

Every irreducible sofic system $S$ has a canonical (see [K]) right resolving cover $\left(R, \pi_{R}\right)$ which intercepts every right closing cover of $S$ [BKM]. (For notational convenience we suppress the dependence on $S$.) This future or right Fischer cover may be constructed as follows: The states of $G(R)$ are the follower sets of magic words of $S$, and for $a$ in the alphabet of $S$ there is an edge labeled $a$ from $F(u)$ to $F(v)$ if and only if $F(v)=F(u a)$. The past or left Fischer cover $\left(L, \pi_{L}\right)$ is obtained by reversing the roles of follower and predecessor.

By the joint cover $\left(J, \pi_{J}\right)$ of $S$ we will mean the unique irreducible component of maximal entropy of the fibered product of $\left(R, \pi_{R}\right)$ and $\left(L, \pi_{L}\right)$. (This fibered product is Krieger's joint finitary state chain $[\mathbf{K}]$.) The joint cover is described by a labeled graph as follows: $G(J)$ has states $(F(u), P(v))$ where $u, v$ are magic words with $u v$ in $S$, and an edge labeled $a$ from $(F(s), P(t))$ to $(F(u), P(v))$ if and only if $F(u)=F(s a)$ and $P(t)=P(a v)$.

If the graph of a cover $(\Sigma, \pi)$ contains a path from state $i$ to state $j$ labeled by the word $w$ of $S$, we say $w$ is an $S$-follower of $i$ terminating at $j$, and also an $S$-predecessor of $j$ originating at $i$. (Unless $\pi$ is right resolving, an $S$-follower of $i$ may terminate at more than one state.) We use the shorthand $i \stackrel{w}{\rightarrow} j$.

Proposition 1.1. The magic $S$-followers [predecessors] of the state $(F(s)$, $P(t))$ in $J$ are exactly the magic words $w$ of $S$ with $P(w)=P(t)[F(w)=F(s)]$.

ProOF. If $(F(s), P(t)) \stackrel{w}{\rightarrow}(F(w), P(x))$ then $P(t)=P(w x)=P(w)$. Conversely, if $P(t)=P(w)$ then $(F(s), P(w))$ is a state of $J$, so this pair is equal to $(F(u), P(v))$ for some magic $u, v$ with $u v$ a word of $S$. Then $s v$ is a word of $S$ since $F(s)=F(u)$, and so $s w$ is a word of $S$ since $P(w)=P(v)$. Let $x$ be a magic word with $s w x$ in $S$; then $P(w)=P(w x)$ and

$$
(F(s), P(w)) \stackrel{w}{\rightarrow}(F(w), P(x)) .
$$

PROPOSITION 1.2. Let $(F(r), P(t))$ and $(F(s), P(t))$ be two states of $J$ with $r$, $s, t$ magic and $F(r) \neq F(s)$. Then

(i) $(F(r), P(t))$ and $(F(s), P(t))$ have no common magic $S$-predecessors, and no common $S$-predecessors originating at the same state.

(ii) $(F(r), P(t))$ and $(F(s), P(t))$ have the same $S$-followers, and their common magic $S$-followers have the same terminal states.

Analogous results hold for states $(F(s), P(t))$ and $(F(s), P(u))$.

PROOF. (i) If $w$ were a common magic $S$-predecessor, by Proposition 1.1 we would have $F(r)=F(w)=F(s)$. If $w$ were a common predecessor originating at $(F(u), P(v))$ we would have $F(r)=F(u w)=F(s)$.

(ii) By Proposition 1.1 these states have the same magic $S$-followers; but every word can be extended forward to a magic word, so they have the same $S$-followers. 
Finally, if $w$ is magic with

$$
(F(r), P(t)) \stackrel{w}{\rightarrow}(F(u), P(v))
$$

then $F(u)=F(w)=F(s w)$ and so

$$
(F(s), P(t)) \stackrel{w}{\rightarrow}(F(u), P(v)) .
$$

2. A characterization of AFT systems. An irreducible sofic system $S$ is said to be almost finite type (AFT) if it admits a cover $\pi$ which is one-to-one on an open set [M]. In fact, if $S$ has such a cover it is conjugate over $S$ to both $\pi_{R}$ and $\pi_{L}$, while if $S$ is not AFT then $\pi_{R}$ and $\pi_{L}$ are not conjugate over $S$ [BKM]. Equivalently, $S$ is AFT if $\pi_{R}$ is left closing (or if $\pi_{L}$ is right closing.) We give another characterization of AFT in terms of the words of $S$.

THEOREM 2.1. For an irreducible sofic system $S$ the following are equivalent:

(i) $S$ is $A F T$.

(ii) If $w$ is a word and $s, s^{\prime}, t, t^{\prime}$ are magic words such that $s w^{i} t, s^{\prime} w^{i} t$ and $s w^{i} t^{\prime}$ are words of $S$ for all $i \geq 0$, then $s^{\prime} w^{i} t^{\prime}$ is a word of $S$ for all sufficiently large $i$.

(Here $w^{i}$ denotes concatenation.)

Proof. Assume $S$ is AFT and let $w, s, s^{\prime}, t, t^{\prime}$ satisfy the hypotheses of (ii). Since $s$ is magic, all appearances of $s w^{i}, s^{\prime} w^{i}$ in $G(R)$ terminate at the states $F\left(s w^{i}\right), F\left(s^{\prime} w^{i}\right)$ respectively. Since $t$ is magic,

$$
F\left(s w^{i} t\right)=F\left(s^{\prime} w^{i} t\right)=F(t)
$$

for all $i$, and the paths labeled $s w^{i} t, s^{\prime} w^{i} t$ must end at this state.

Now, since $\pi_{R}$ is left closing, for some positive integer $N$ all paths with the same labeling and terminal state coincide from the $N$ th state on. Applying this to paths labeled $w^{i} t$ we see that for all sufficiently large $i$,

$$
F\left(s w^{i}\right)=F\left(s^{\prime} w^{i}\right)
$$

But $t^{\prime} \in F\left(s w^{i}\right)$, so $s^{\prime} w^{i} t^{\prime}$ is a word in $S$ for all sufficiently large $i$.

Conversely, suppose $S$ is not AFT. Then $\pi_{L}$ is not conjugate to the joint cover $\pi_{J}$, for if it were, then $\pi_{R}$ would intercept $\pi_{L}$, making $\pi_{R}$ left closing by Lemma 12 of [BKM]. So the projection of $J$ onto $L$ must collapse a pair of periodic points. Let $v^{\infty}$ be the image of these points in $S$, with $v$ aperiodic. Then in $J$ we have

$$
\begin{aligned}
& (F(s), P(t)) \stackrel{v^{l}}{\rightarrow}(F(s), P(t)), \\
& \left(F\left(s^{\prime}\right), P(t)\right) \stackrel{v^{m}}{\rightarrow}\left(F\left(s^{\prime}\right), P(t)\right)
\end{aligned}
$$

for some $l, m \in N$ and magic $s, s^{\prime}, t$ with $F(s) \neq F\left(s^{\prime}\right)$. The words $s v^{i l} t$ and $s^{\prime} v^{i m} t$ are in $S$ for all $i \geq 0$.

We may assume, interchanging $s$ and $s^{\prime}$ if necessary, that there is a magic word $u \in F(s) \backslash F\left(s^{\prime}\right)$. Then $u$ is not an $S$-follower of $\left(F\left(s^{\prime}\right), P(t)\right)$, so by Proposition $1.2, u$ cannot follow $(F(s), P(t))$ either. But $J$ must have a state $(F(s), P(u))$ with $S$-predecessor $s$ and $S$-follower $u$. 
For every $i \geq 0, s v^{i l}$ is an $S$-predecessor of $(F(s), P(t))$, so by Proposition 1.2 it precedes $(F(s), P(u))$ as well, and $s v^{i l} \in P(u)$. The collection of states $\left\{P\left(v^{i l} u\right): i \geq 0\right\}$ must be finite, so there are nonnegative integers $p, N$ such that

$$
P\left(v^{i l} u\right)=P\left(v^{(i+p) l} u\right)
$$

for $i \geq N$. Set $k=p l$ and $t^{\prime}=v^{j l} u$ where $j \geq N$ is a multiple of $m$. Then $P\left(v^{k} t^{\prime}\right)=P\left(t^{\prime}\right)$, and $F\left(s v^{k}\right)=F(s)$ from $(*)$. So in $J$,

$$
\left(F(s), P\left(t^{\prime}\right)\right) \stackrel{\boldsymbol{v}^{k}}{\rightarrow}\left(F(s), P\left(t^{\prime}\right)\right) .
$$

Finally, set $w=v^{n}$ where $n=\operatorname{lcm}(m, k)$. Then for all $i \geq 0, s w^{i} t, s^{\prime} w^{i} t$ and $s w^{i} t^{\prime}$ are words of $S$. Now

$$
s^{\prime} w^{i} t^{\prime}=s^{\prime} v^{i n} v^{j l} u=s^{\prime} v^{c m} u
$$

for some $c$. But $F\left(s^{\prime} v^{c m}\right)=F\left(s^{\prime}\right)$ and $u$ is not in this set, so $s^{\prime} w^{i} t^{\prime}$ is not a word of $S$.

REMARK 2.2. Note that in the proof of "not (i) implies not (ii)" we obtained a slightly stronger result, since $s^{\prime} w^{i} t^{\prime}$ fails to be in $S$ for every $i$, not just for sufficiently large $i$. We will use this stronger form in the proof of Theorem 4.1.

REMARK 2.3 Osikawa [0] has recently given a related characterization of AFT in terms of configurations of paths in the Fischer cover.

3. Some constructions of extensions of SFT. Let $\Sigma$ be a SFT. In what follows, it will be convenient to assume that the graph $G=G(\Sigma)$ has at most one edge from $i$ to $j$ for all vertices $i, j$. (We can achieve this by passing to a higher block presentation.)

By a cycle of length $l$ in $G$ we mean a sequence $\alpha=\alpha_{1} \cdots \alpha_{l}$ of vertices of $G$ with an edge from $\alpha_{l}$ to $\alpha_{1}$ and from $\alpha_{i}$ to $\alpha_{i+1}$ for $1 \leq i<l$. The cycle $\alpha$ is simple if the $\alpha_{i}$ are distinct, and we will call it supersimple if in addition there is no edge from $\alpha_{i}$ to $\alpha_{j}$ for $j \not \equiv i+1$ modulo $l$.

The $n$-block presentation $\Sigma^{(n)}$ of $\Sigma$ is given by a directed graph $G^{(n)}$ which has as its vertices the $n$-blocks of $\Sigma$, with an edge from $\left(i_{1} \cdots i_{n}\right)$ to $\left(i_{2} \cdots i_{n+1}\right)$ if $G$ has an edge from $i_{n}$ to $i_{n+1}$. We denote by $\alpha^{(n)}$ the cycle in $G^{(n)}$ with vertices

$$
\alpha_{i}^{(n)}=\alpha_{i} \alpha_{i+1} \cdots \alpha_{i+n-1}, \quad 1 \leq i \leq l,
$$

where the addition is modulo $l$. Note that if $\alpha$ is simple then $\alpha^{(2)}$ is supersimple.

The following appear in $[\mathbf{A M}]$ as Propositions 3.3 and 3.4.

PROPOSITION 3.1. If $\alpha$ is a simple cycle, so is $\alpha^{(n)}$ for all $n$. If $\alpha$ is an aperiodic cycle of length $l$ then $\alpha^{(l)}$ is simple.

PROPOSITION 3.2. Let $\alpha, \beta$ be cycles in $G$ of lengths $k, l$ and let $m$ be the length of the largest common subblock of $\alpha^{\infty}$ and $\beta^{\infty}$. If $m<\min (k, l)$ then $\alpha^{(n)}$ is disjoint from $\beta^{(n)}$ for $n \geq m+1$.

We now describe two techniques for constructing a resolving, one-to-one a.e. cover $\bar{\Sigma}$ of a SFT $\Sigma$. Essentially the same constructions appear in the proof of the covering lemma of $[\mathbf{B}]$.

Construction 3.3. Suppose $\alpha=\alpha_{1} \cdots \alpha_{l}$ is a supersimple cycle of the graph $G$ of $\Sigma$ and let $A$ denote the set of edges entering $\alpha$ (that is, with terminal, but not 
initial, vertex on $\alpha$.) Suppose $\{B, C\}$ is a partition of $A$ into nonempty sets. We obtain a directed graph $\bar{G}$ from $G$ as follows: The cycle $\alpha$ is replaced by two new disjoint supersimple cycles $\beta=\beta_{1} \cdots \beta_{l}$ and $\gamma=\gamma_{1} \cdots \gamma_{l}$. If $e$ is an edge in $A$ with terminal vertex $\alpha_{i}$, we replace it in $\bar{G}$ by an edge with the same initial vertex and terminal vertex $\beta_{i}$ or $\gamma_{i}$, according as $e \in B$ or $e \in C$. An edge from $\alpha_{i}$ to $j, j$ not on $\alpha$, is replaced by edges from $\beta_{i}$ to $j$ and from $\gamma_{i}$ to $j$.

We say that $\bar{G}$ and the associated SFT $\bar{\Sigma}$ are obtained by a cycle splitting by entry with cycle $\alpha$ and partition $\{B, C\} . \bar{\Sigma}$ is a right resolving cover of $\Sigma$ (with each new edge mapped to the one it replaced.) Cycle splitting by departure is defined analogously, and yields a left resolving cover.

Construction 3.4. Suppose $\alpha=\alpha_{1} \cdots \alpha_{l}$ is a supersimple cycle of $G$ and let $m$ be a positive integer. We form a new graph $\bar{G}$ in which $\alpha$ is replaced by a new supersimple cycle $\beta=\beta_{1} \cdots \beta_{m l}$ of length $m l$. Each edge of $G$ from $j$ to $\alpha_{i}, j$ not on $\alpha$, is replaced in $G$ by an edge from $j$ to $\beta_{i}$. Each edge from $\alpha_{i}$ to $j, j$ not on $\alpha$, is replaced by $m$ edges, one from $\beta_{i+k l}$ to $j$ for $0 \leq k \leq m-1$.

We say the graph $\bar{G}$ and associated SFT $\bar{\Sigma}$ are obtained from $G$ and $\Sigma$ by period multiplication (of $\alpha$ by $m$ ) with fixed entry. $\bar{\Sigma}$ is a right resolving cover of $\Sigma$ via the map which sends new edges to those they replaced. Period multiplication with fixed departure is defined analogously and yields a left resolving cover.

REMARK 3.5. If $\Sigma$ is a cover of the sofic system $S$ via an edge labeling of the graph $G$, then in each of the above constructions we can give each new edge of $G$ the label of the edge it replaced; this makes $\bar{\Sigma}$ a cover of $S$ which is intercepted by the cover $\Sigma$.

4. Covers with no common interceptors. We are now ready to prove our main theorem.

THEOREM 4.1. Let $S$ be an irreducible sofic system which is not almost finite type. Then there exists an infinite family of covers of $S$ such that no two are intercepted by a common cover of $S$.

There are three steps to the proof.

(i) SIMPLIFYING THE PICTURE. By Theorem 2.1 and Remark 2.2, $S$ contains a word $w$ and magic words $s, s^{\prime}, t, t^{\prime}$ such that for all $i \geq 0, s w^{i} t, s^{\prime} w^{i} t$ and $s w^{i} t^{\prime}$ are words of $S$ but $s^{\prime} w^{i} t^{\prime}$ is not. In fact, the proof of Theorem 2.1 shows that the joint cover $\left(J, \pi_{J}\right)$ has distinct states $\alpha_{1}=\left(F(s), P\left(t^{\prime}\right)\right), \beta_{1}=(F(s), P(t))$ and $\gamma_{1}=\left(F\left(s^{\prime}\right), P(t)\right)$, and cycles $\alpha, \beta$ and $\gamma$ based at these states which are labeled

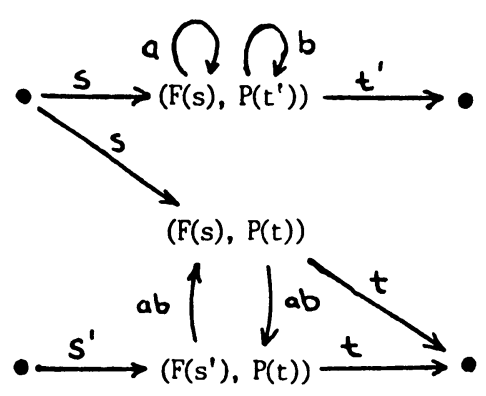

FIGURE 1

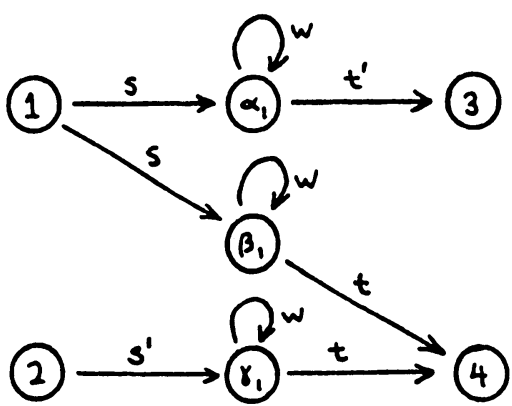

FIGURE 2 


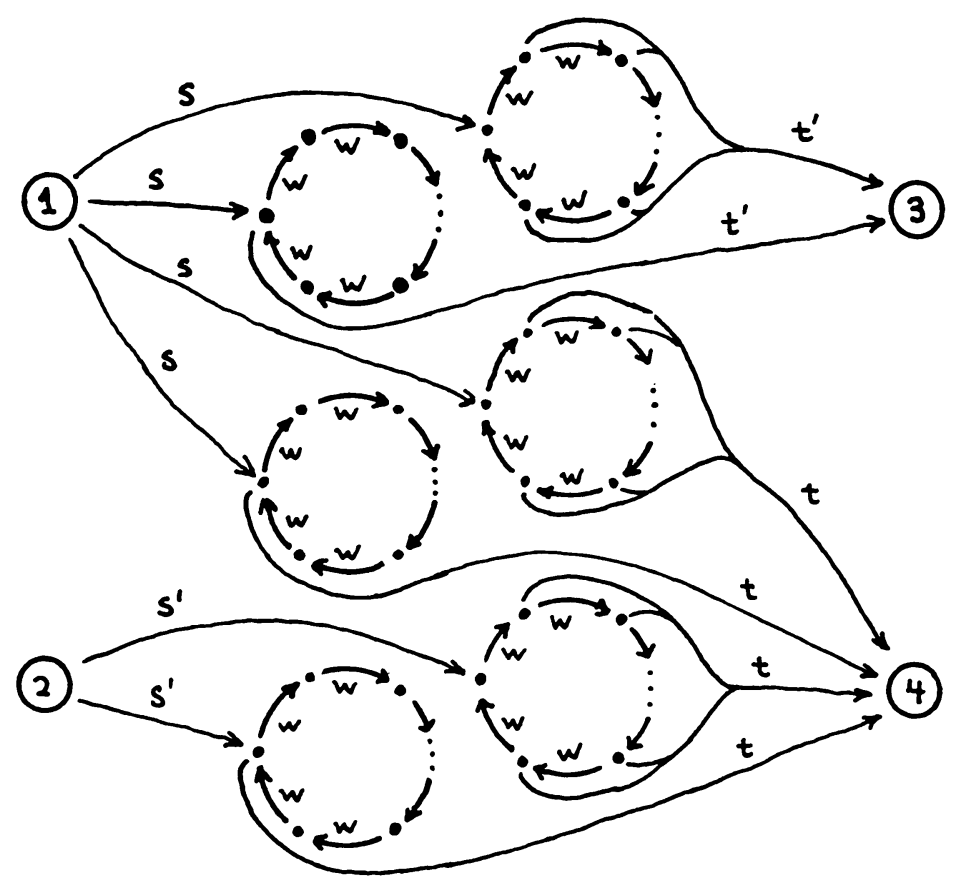

FIGURE 3

by $w$. In Figure 1 we give an illustrative example with $w=a b a b$. By Proposition 1.2 , the paths labeled $s$ preceding $\alpha_{1}$ and $\beta_{1}$ have a common initial state, and those labeled $t$ following $\beta_{1}$ and $\gamma_{1}$, a common terminus.

Passing to a higher block presentation of $J$ if necessary, we can assume (by Proposition 3.1) that each of the cycles $\alpha, \beta, \gamma$ has the form $\xi^{j}$ for some supersimple cycle $\xi$, and (by Proposition 3.2) that these cycles are pairwise either disjoint or cyclic permutations of one another. (When we move to the higher block presentation we must modify the words $s, s^{\prime}, t, t^{\prime}$, extending them so that, for example, the paths labeled $s$ still have the same initial vertex and still enter the cycles $\alpha$ and B.)

Now we construct a one-to-one a.e. extension $\bar{J}$ of $J$ in which these cycles are replaced by new ones-which we will continue to call $\alpha, \beta, \gamma$-that are distinct (use cycle splitting, Construction 3.3), are supersimple (if $\alpha=\xi^{j}$, use period multiplication of $\xi$ by $j$, Construction 3.4), and have no entering or departing edges except those on the paths labeled $s$ or $s^{\prime}, t$ or $t^{\prime}$ which enter and leave states $\alpha_{1}, \beta_{1}, \gamma_{1}$ (more cycle splitting). Thus the graph $G(\bar{J})$ contains the configuration in Figure 2. By standard state-splitting techniques we can now arrange for the rest of $G(J)$ to meet this configuration only in the states $1,2,3$, and 4 . (These states need not be distinct.)

(ii) CONStructing the COVERS. We now imitate the construction in [W]. For a fixed positive integer $m$ we form a new labeled graph $G\left(\bar{\Gamma}_{m}\right)$ by replacing the configuration of Figure 2 in $G(\bar{J})$ by that of Figure 3. 


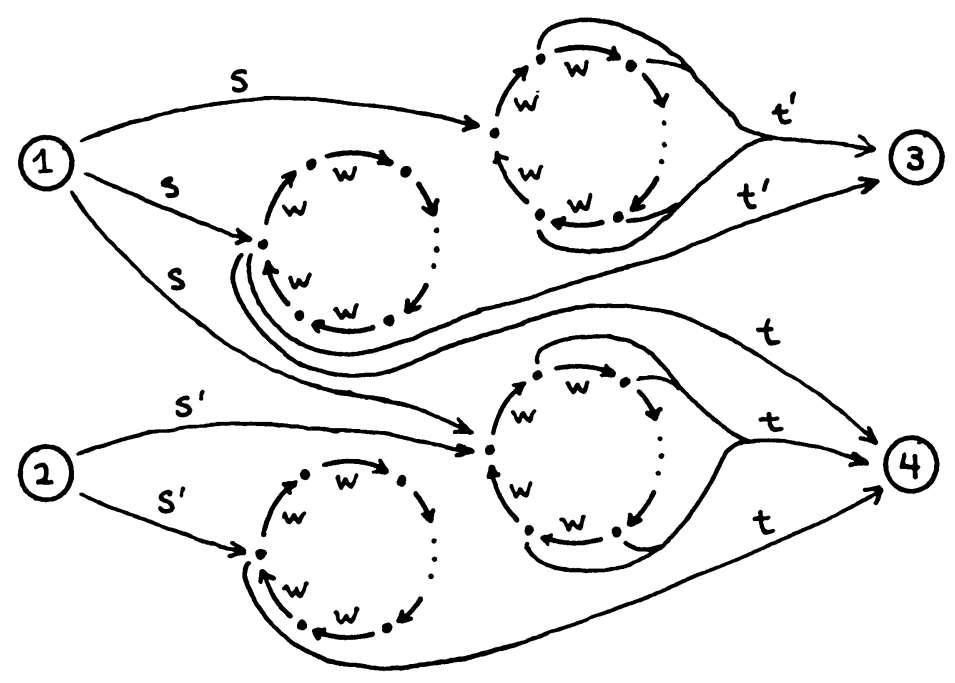

FIGURE 4

We have first multiplied the periods of $\alpha, \beta$, and $\gamma$ by $m$, keeping the entries fixed; the resulting cycles each have $m$ departing paths labeled $t$ or $t^{\prime}$. Next we have split each of these cycles into two by partitioning the departing edges so that one cycle keeps the edge departing from the base while the other keeps the remaining $m-1$ departing edges. The resulting SFT $\bar{\Gamma}_{m}$ is a one-to-one a.e. cover of $S$.

Finally, we collapse the second and fourth cycles from the top in Figure 3 to a single cycle $\delta(m)$, and also collapse the third and fifth cycles to a cycle $\varepsilon(m)$, as shown in Figure 4. These moves are the inverses of cycle splittings, by departure in the first instance and by entry in the second. The resulting SFT $\Gamma_{m}$ is not an extension of $J$, but it is a one-to-one a.e. cover of $S$.

We claim that the covers $\Gamma_{m}$ for $m=1,2, \ldots$ meet the conditions of the theorem.

(iii) VERIFICATION. Suppose to the contrary that two covers $\Gamma_{m}, \Gamma_{n}$ with $m>n$ are intercepted by a cover $(\Sigma, \pi)$ :

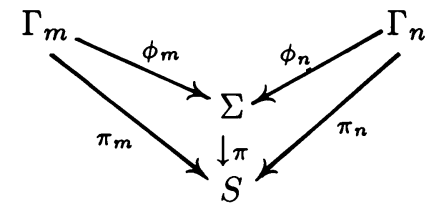

We may assume $\pi$ is given by an edge labeling of a directed graph $G(\Sigma)$.

Let $x$ be a resolving word for $\pi$ which labels a path in $\bar{J}$ from state 4 to state 1 in Figure 2. (If these states are the same we must avoid choosing $x=s w^{i} t$.) Then for every $i,\left(s w^{i} t x\right)^{\infty}$ is an orbit of $S$ which has a unique preimage of the same period in $\Sigma$, and also in $\Gamma_{m}$ and $\Gamma_{n}$. If $\operatorname{lcm}(m, n)$ divides $i$ then the preimage in $\Gamma_{m}$ passes through the cycle $\delta(m)$, and the preimage in $\Gamma_{n}$ passes through $\delta(n)$. Hence the periodic orbits generated by the cycles $\delta(m), \delta(n)$ must have a common image in $\Sigma$, generated by a cycle $\delta$ of period dividing $|w| \cdot \operatorname{gcd}(m, n)$.

Now for large $i$ the preimage of $\left(s w^{i} t x\right)^{\infty}$ in $\Sigma$ must pass through $\delta$ not only when $\operatorname{lcm}(m, n)$ divides $i$, but whenever $\operatorname{gcd}(m, n)$ divides $i$. But the preimage in 
$\Gamma_{m}$ passes through $\varepsilon(m)$ if $m$ does not divide $i$. So $\phi_{m}$ must take the cycle $\varepsilon(m)$ to $\delta$.

In a similar fashion we find words $y, z$ for which $\left(s w^{i} t^{\prime} y\right)^{\infty}$ and $\left(s^{\prime} w^{j} t z\right)^{\infty}$ are orbits in $S$ with unique preimages in all three covers. If $m$ divides $i$ but not $j$, the preimages in $\Gamma_{m}$ pass through $\delta(m)$ and $\varepsilon(m)$ respectively, so if $i$ and $j$ are also sufficiently large both preimages in $\Sigma$ pass through $\delta$. Splicing the paths of these orbits where they pass through $\delta$, we find there is a path in $G(\Sigma)$ labeled by $s^{\prime} w^{k} t^{\prime}$ for some $k$. Since this is not a word of $S$, we have a contradiction.

\section{REFERENCES}

[AM] R. Adler and B. Marcus, Topological entropy and equivalence of dynamical systems, Mem. Amer. Math. Soc., no. 219, 1979.

[B] M. Boyle, Lower entropy factors of sofic systems, Ergodic Theory and Dynamical Systems 4 (1984), 541-557.

[BKM] M. Boyle, B. Kitchens and B. Marcus, A note on minimal covers for sofic systems, Proc. Amer. Math. Soc. 95 (1985), 403-411.

[BMT] M. Boyle, B. Marcus and P. Trow, Resolving maps and the dimension group for shifts of finite type, Mem. Amer. Math. Soc. no. 377, 1987.

[K] W. Krieger, On sofic systems. I, Israel J. Math. 48 (1984), 305-330.

[M] B. Marcus, Sofic systems and encoding data, IEEE-IT 31 (1985), 366-377.

[O] M. Osikawa, A classification of transitive sofic systems, preprint.

[W] S. Williams, A sofic system with infinitely many minimal covers, Proc. Amer. Math. Soc. 98 (1986), 503-506.

Department of Mathematics and Statistics FCS \#3, UNiversity of South Alabama, Mobile, Alabama 36688 